\title{
Transmission and radiation of an accelerating mode in a photonic band-gap fiber
}

\author{
C.-K. Ng, R. J. England, L.-Q. Lee, R. Noble, V. Rawat, and J. Spencer \\ SLAC National Accelerator Laboratory, 2575 Sand Hill Road, Menlo Park, California 94025, USA
}

(Received 7 July 2010; published 6 December 2010)

\begin{abstract}
A hollow-core photonic band-gap (PBG) lattice in a dielectric fiber has been proposed as a highgradient low-cost particle accelerator operating in the optical regime where the accelerating mode confined to a defect in the PBG fiber can be excited by high-power lasers [X. Lin, Phys. Rev. ST Accel. Beams 4, 051301 (2001)]. Developing efficient methods of coupling laser power into these structures requires a thorough examination of the propagating mode and its near and far-field radiation. In this paper, we develop a simulation method using the parallel finite-element electromagnetic suite ACE3P to calculate the radiation of the propagating accelerator mode into free space at the end of the fiber. The far-field radiation will be calculated and the mechanism of coupling power from an experimental laser setup will be discussed.
\end{abstract}

DOI: 10.1103/PhysRevSTAB.13.121301

PACS numbers: 41.75.Jv, 42.70.Qs

\section{INTRODUCTION}

Because of electrical breakdown of metals in the presence of high electric fields, traditional particle accelerator structures, which consist of metal cavities driven by highpower microwaves, typically cannot generate accelerating fields greater than 50 to $100 \mathrm{MV} / \mathrm{m}$. By comparison, the surface fields of dielectric materials exposed to pulsed laser light can exceed this limit by 1 to 2 orders of magnitude, making a laser-powered optical waveguide an attractive medium for particle acceleration [1-3]. The simplest such scheme, a hollow dielectric tube [1], requires a conducting outer boundary to confine the fields, making it unsuitable for optical frequencies where the Ohmic losses in metals are prohibitive. An alternative is to replace the conducting boundary with layers of dielectric material of alternating refractive index, thereby forming a dielectric Bragg reflector which can confine an accelerating mode [2]. More recently, it has been proposed to use a glass fiber permeated by a lattice of vacuum holes surrounding a central "defect" hole of larger diameter [3]. The lattice forms a photonic band-gap (PBG) crystal, with the operating wavelength of the accelerator lying inside the band gap. The defect hole acts as both the region where the accelerating fields are confined and as a vacuum channel for the accelerated particles.

Despite its more complex geometry (seen in a quarter cross section in Fig. 1), the PBG fiber of Ref. [3] has several notable benefits over the Bragg fiber of Ref. [2]: the diameter of the central beam channel is approximately a factor of 2 larger for the same operating wavelength and accelerating gradient; the structure is made entirely out of a single material, avoiding difficulties inherent in combining materials of different mechanical and thermal properties; and fabrication techniques for constructing these fibers are well established, making custom prototype construction a realistic near-term goal. As discussed in Ref. [2], when used as particle accelerators, such fibers can in principle achieve high gradients, operating in the optical regime using lasers for excitation of the accelerating mode. However, the coupling of laser power to the PBG fiber has not been previously addressed.

This paper will focus on numerical simulations of the propagation of the defect mode in the PBG fiber and from the fiber into free space. These studies are required to inform planned experimental efforts to excite prototype fibers with lasers and measure the resultant acceleration of an electron beam [4]. The radiation pattern in the far field is of particular interest, because it represents (when propagated in reverse) the ideal laser profile for optimal

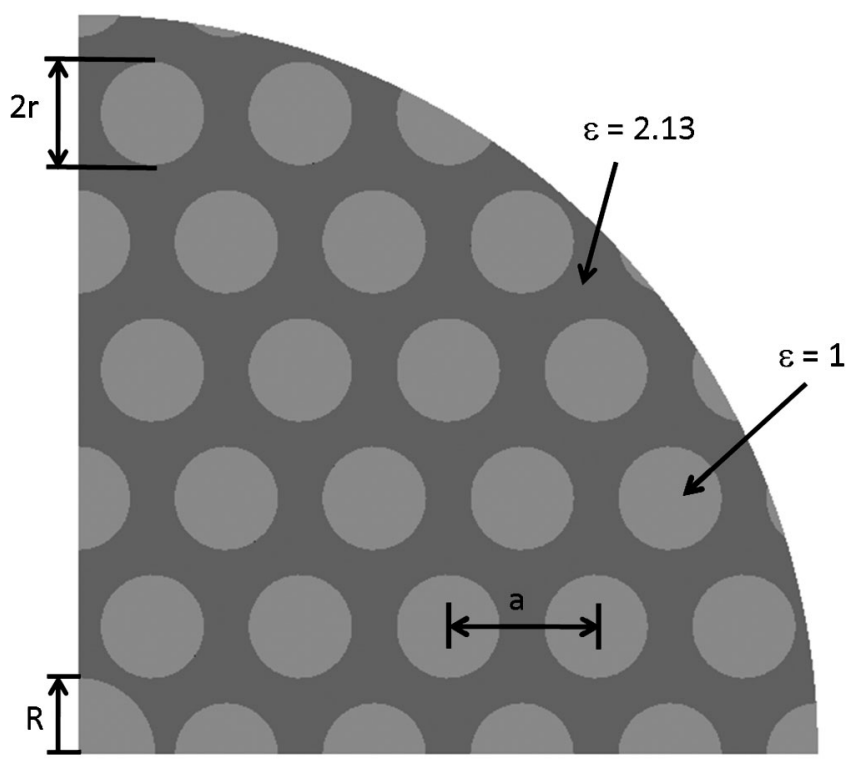

FIG. 1. One quarter of the Lin PBG structure, consisting of a lattice of vacuum holes (white) in a glass substrate (gray) with hole-to-hole spacing $a$ and radii $r=0.35 a$. The mode propagates in the central defect hole of radius $R=0.52 a$. 
coupling to the structure. We use the electromagnetic simulation package ACE3P [5] developed at SLAC for the simulations. ACE3P is a suite of parallel electromagnetic codes based on the finite-element method, and it consists of solvers in both the frequency and time domains. There are several advantages of using these codes for our simulations. First, tetrahedral finite elements with quadratic curved surfaces are well suited for modeling the curved geometries of the PBG fiber with high fidelity. The finite elements also have higher-order basis function representations which allow solutions with high accuracy. Second, the simulation of the propagation of the accelerating mode requires its excitation at a port boundary of the computational volume. The electromagnetic field pattern of the defect mode can be solved by the frequency-domain eigensolver OMEGA3P [6] in ACE3P, and then loaded into the time-domain solver T3P [7], where appropriate boundary conditions can be imposed on the outer surfaces of the computational volume to terminate the propagation of electromagnetic waves. Third, the parallel capability of these codes allows large problems to be solved on parallel computers. This is useful for simulating the radiation into free space from the accelerating mode by using a large computational volume.

This rest of the paper is organized as follows. In Sec. II, we discuss the background and motivation for this work. In Sec. III, we use the frequency-domain eigensolver OMEGA3P to determine the defect mode in the PBG fiber. In Sec. IV, we describe a numerical procedure to simulate the propagation of the defect mode traveling in the PBG fiber using the time-domain code T3P. In Sec. V, we calculate the power radiated from the accelerating mode into free space and determine the far-field radiation pattern. A summary of the results is given in the last section.

\section{BACKGROUND}

A hollow-core, photonic band-gap (PBG) fiber is drawn as a two-dimensional periodic dielectric array that is uniform longitudinally, so it acts like a waveguide along which the light propagates in a central defect region. The transverse periodic dielectric structure actually serves two essential purposes. It both slows the wave phase velocity in the longitudinal propagation direction and provides a transverse confinement mechanism so the mode remains trapped in the central region where particle acceleration occurs. The confinement is due to the fact that solutions to Maxwell's equations in a periodic system must exhibit the underlying symmetry of the periodic array, which results in passbands and stop bands through constructive and destructive interference. Modes that would correspond to frequencies in the stop band (or photonic band gap in optics terminology) are not allowed to propagate in the perfect lattice but trapped, slow-wave modes may result when the symmetry of the dielectric array is broken by introduction of a central defect hole.
The PBG accelerator idea was introduced by Kroll et al. [8], who proposed using a transverse array of dielectric rods (relative permittivity $=9$ ) in air, separated by periodic conducting plates in the longitudinal direction. The central rod was removed to introduce an open defect, leaving space for a particle beam to propagate. The rods provided the transverse mode confinement and the conducting plates permitted a selection of the wave number, and hence phase velocity for a transverse magnetic (TM) mode in the defect region. In the work of Kroll et al., PBG structures at $11 \mathrm{GHz}(1.5 \mathrm{~cm}$ lattice period) were fabricated, and the presence of confined modes was confirmed.

In the present work, we investigate the accelerating mode propagation, radiation, and coupling properties in a different PBG structure made completely with dielectric and consisting of a periodic hexagonal array of holes in a glass matrix, which was first explored by Lin [3]. Based on a number of calculations, fabrication experiments, and radiation damage studies, we expect that a PBG accelerator should be capable of gradients of some 20-100 times that of a conventional $\mathrm{rf}$ or superconducting accelerators [9]. For these structures, currents on the order of $10^{11}$ electrons per pulse should be possible from a matrix cathode measuring $1 \mathrm{~mm}^{2}[10,11]$.

It is also important that the power coupled into the PBG fiber will propagate in the forward direction along the beam axis using the traveling-wave defect mode for acceleration. In order to understand the complexities involved in designing a power coupler for the PBG fiber, it is instructive to study both the transmission and radiation pattern from the propagation of the accelerating mode in the PBG fiber through numerical simulation. This will provide insights for the mechanism of exciting the accelerating mode in an experimental setup. This is stated in the Conclusion.

\section{DEFECT MODE IN PHOTONIC BAND-GAP FIBER}

The PBG fiber (see Fig. 1) proposed by Lin consists of circular holes in a hexagonal lattice with spacing $a$ between the centers of neighboring holes. The holes have a radius of $0.35 a$. In order to introduce a defect in the lattice, one hole is replaced by another with a radius of $0.52 a$. The material of the fiber has a dielectric constant of 2.13, corresponding to glass at near IR wavelengths. In Lin's paper, the defect mode is obtained using the plane wave method [12] with the propagation constant $\beta=k_{z} / k_{0}=$ 1 , where $k_{0}$ and $k_{z}$ are the free-space wave number and its component along the propagation $z$ axis, respectively. Here we used OMEGA3P, a Maxwell eigensolver that was developed to solve for the resonant modes of an accelerator cavity. The simulation uses a thin slab of the PBG fiber with circular cross section, which is truncated at five lattice constants from the center of the defect. By imposing different boundary conditions at opposite sides of the slab, the frequencies of standing wave modes are obtained with their 


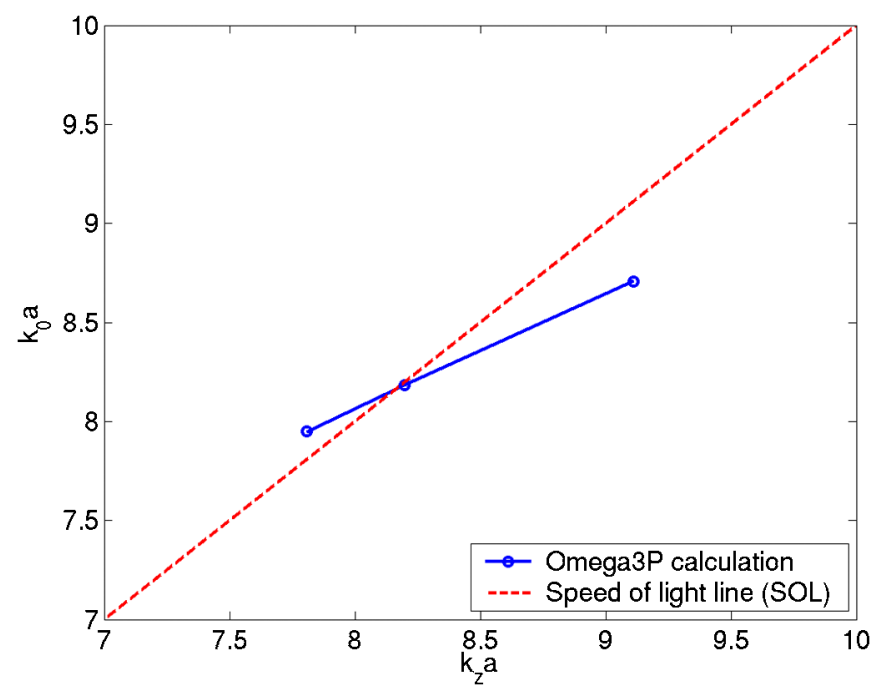

FIG. 2. Dispersion curve calculated by OMEGA3P near the PBG defect mode.

wavelengths determined by the slab thickness. OMEGA3P solves for a number of modes above a certain specified frequency, and the defect mode can be identified from the field patterns of these modes. By repeating the simulation for different slab thicknesses, the frequency of the defect mode as a function of the propagation constant is obtained. The dispersion curve is shown in Fig. 2, from which the frequency of the synchronous mode propagating at the speed of light is found to be $8.2 c / a$, the same as that obtained in Ref. [3]. For example, the choice of $a=2.61 \mu \mathrm{m}$ would yield an accelerating mode with a wavelength $\lambda=2 \mu \mathrm{m}$.

The longitudinal and transverse electric field patterns and the transverse magnetic field pattern are shown in Fig. 3. All relevant figures in this paper use a rainbow color scheme to represent magnitude with the red and blue being the maximum and minimum, respectively. It can be seen from Fig. 3(a) that the defect mode is TM-like with a uniform distribution of longitudinal field inside the defect hole that can be used for acceleration. All the field components exhibit the sixfold symmetry of the lattice, with their maxima located at distances $0.8-1.5 a$ from the center

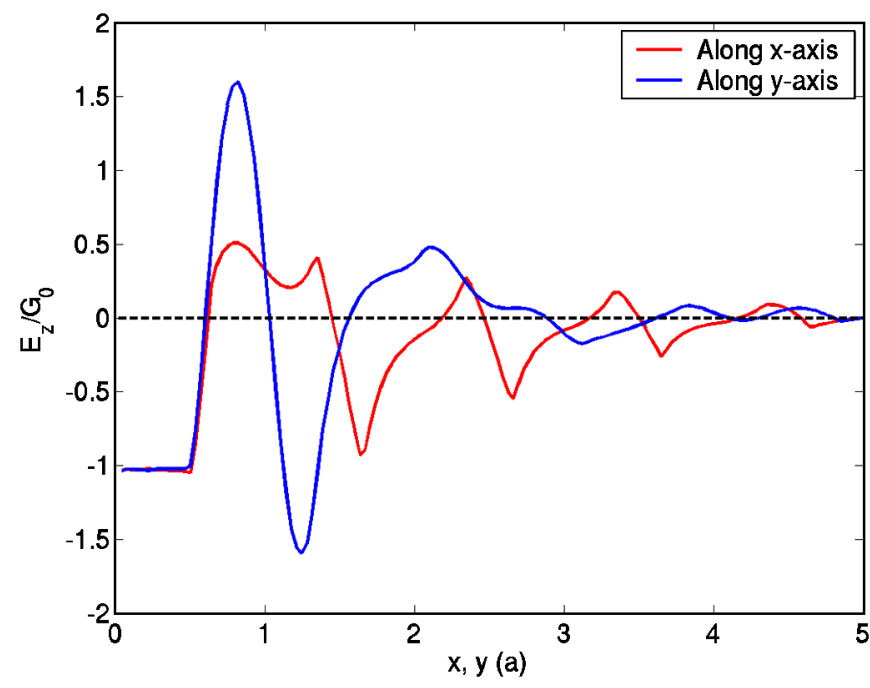

FIG. 4. Variations of the longitudinal electric field normalized to axial gradient of the defect mode along the $x$ axis and the $y$ axis. Note that the field distribution is uniform at distances inside the defect region $(x, y<R=0.52 a)$.

of the defect. The values of the transverse electric and magnetic fields at different locations of the cross section of the PBG fiber obtained from OMEGA3P are written to files which will then be read in as inputs to the time-domain solver T3P for studying mode propagation (Sec. IV). It is instructive to see how the defect mode is confined by studying the decay of its electromagnetic fields from the center of the defect hole. Figure 4 shows the variations of the longitudinal component of the electric field as a function of the transverse position (normalized to $a$ ) along the $x$ axis and the $y$ axis. The field decays to less than a few $\%$ of the maximum when the distance is greater than $4 a$ in both directions. In the defect hole, the longitudinal field remains essentially constant, as desired for an acceleration mode.

Note that regions of higher field exist outside the defect as seen in Figs. 3 and 4. The ratio $D F$ of peak field outside of the defect to axial accelerating field determines the maximum achievable unloaded gradient $G_{0}$ in the structure via $G_{0}=E_{\max } / D F$, where $E_{\max }$ is the damage threshold of the material. For this structure, the peak field lies off axis
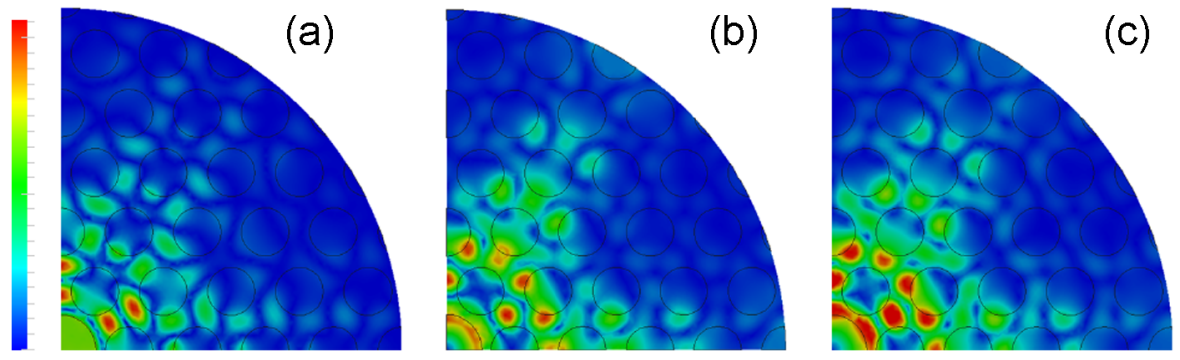

FIG. 3. Field magnitude of the defect mode in the PBG fiber: (a) longitudinal electric field; (b) transverse electric field; (c) transverse magnetic field. Note that a rainbow color scheme (the legend) is used to represent magnitude with the red and blue colors being the maximum and minimum, respectively. This color scheme will be used throughout the paper for all color plots. 
at an azimuthal angle of 30 degrees, giving $D F=2$. For a laser pulse length of 1 ps operating at $\lambda=2 \mu \mathrm{m}$, the damage fluence of glass is approximately $2 \mathrm{~J} / \mathrm{cm}^{2}$ [13], which translates to an approximate field magnitude $E_{\max } \approx$ $5 \mathrm{GV} / \mathrm{m}$ in the structure. Hence, the maximum gradient in this example is $G_{0} \approx 2.5 \mathrm{GV} / \mathrm{m}$.

\section{PROPAGATION OF DEFECT MODE}

We use the time-domain code T3P to study the propagation of the defect mode in the PBG fiber. Consider a slab of the PBG fiber with more than $2 \lambda$ thickness as shown in Fig. 5, where $\lambda$ is the wavelength of the source. The ends of the slab are treated as waveguide ports such that the defect mode propagates from the input port on the left, transits through the slab, and exits at the output port on the right. The transverse components of the electric and magnetic fields for the defect mode obtained using OMEGA3P in the previous section are loaded at the input port as an external excitation in T3P. In order to minimize excitations of frequency contents other than the defect mode frequency, a narrowband pulse with a slow rise time is driven at the input port. Typically, a rise time of $20-30$ periods is sufficient to maintain the monochromatic feature of the excitation. Absorbing boundary conditions [7] are imposed at both the input and output ports to terminate any electromagnetic field propagation so that reflection back to the computational volume is minimized. The time-domain simulation is carried out until steady state is reached. Figures 5(a) and 5(b) show the electric field pattern during the rise time of the driven pulse and that at steady state, respectively. It can be seen that the steady state field demonstrates the establishment of a traveling mode in the PBG fiber.

The instantaneous power at the input and output ports is determined by integrating the Poynting flux over the areas

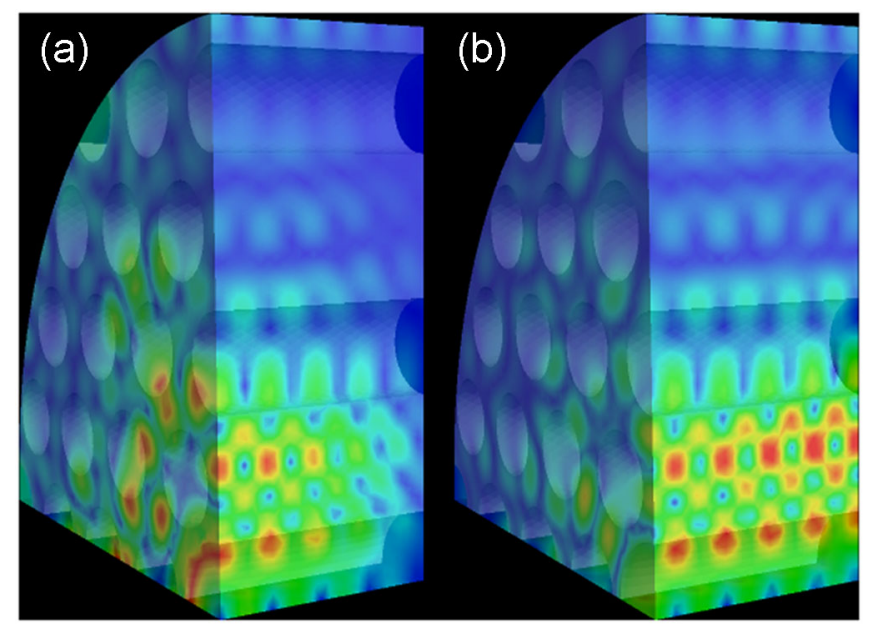

FIG. 5. Snapshots of electric field amplitude for propagation of defect mode in fiber slab: (a) during transient; (b) at steady state. In (b), one again sees the uniform distribution in the defect.

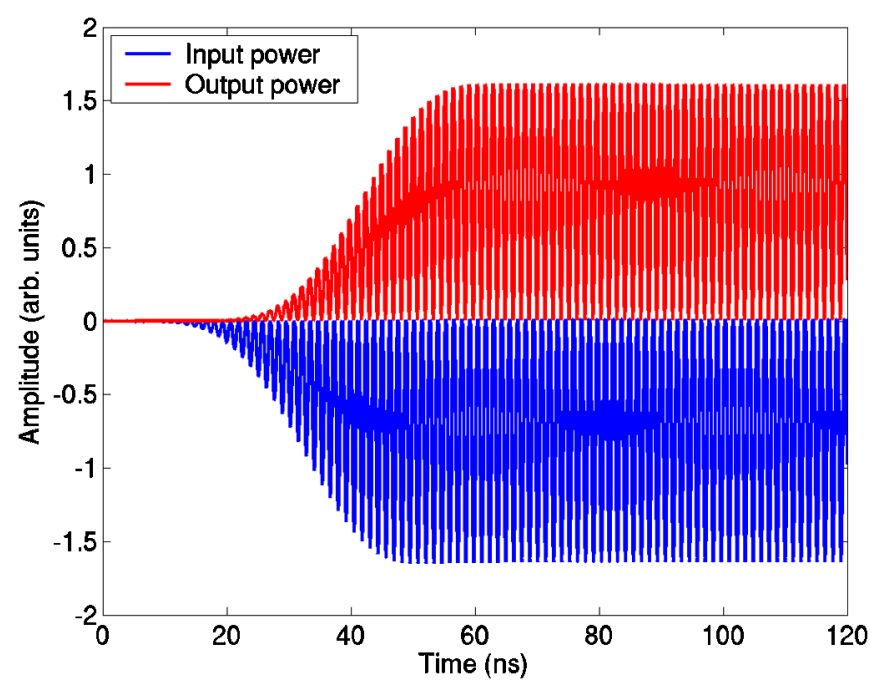

FIG. 6. Power transmission in the PBG fiber slab. The blue and red curves represent the input and output pulse, respectively.

of the ports. Figure 6 shows the power as a function of time at these ports. The positive and negative values of the power indicate net power flow out of and into the computational volume, respectively. At the input port, the evolution of the power follows that of the driven pulse and the power propagates in the forward direction into the PBG fiber slab. The power at the output end has a similar pattern as that at the input port, and its magnitude is the same (with less than $1 \%$ difference) but of opposite sign as that of the excitation at the input port, as expected. There is a time delay between the input and output signals determined by the group velocity of the traveling wave, which is found to be $0.6 c$, in agreement with that obtained in Ref. [3].

\section{SIMULATION OF RADIATION FROM DEFECT MODE}

\section{A. Radiation from defect mode}

To provide insights of how to couple power into the PBG fiber, it is instructive to study the inverse problem first, namely, the radiation pattern from the propagation of the defect mode into free space. The radiation pattern will provide guidelines to project laser beams near the end of the PBG fiber to excite the defect mode. To this end, we use the time-domain code T3P to simulate the model as shown in Fig. 7. Because of symmetry, only one quarter of the PBG fiber is simulated. In this model, a PBG slab is placed in a spherical volume whose spherical surface is terminated using an absorbing boundary condition so that the outgoing waves diffracted at the interface between the PBG fiber and the vacuum will not be reflected back into the computational volume. In a similar manner as discussed in the previous subsection, the defect mode is excited at the input port located at the left end of the PBG slab, propagates through the slab, and then radiates into free space. Again, to maintain the monochromatic feature of the pulse, a slow 


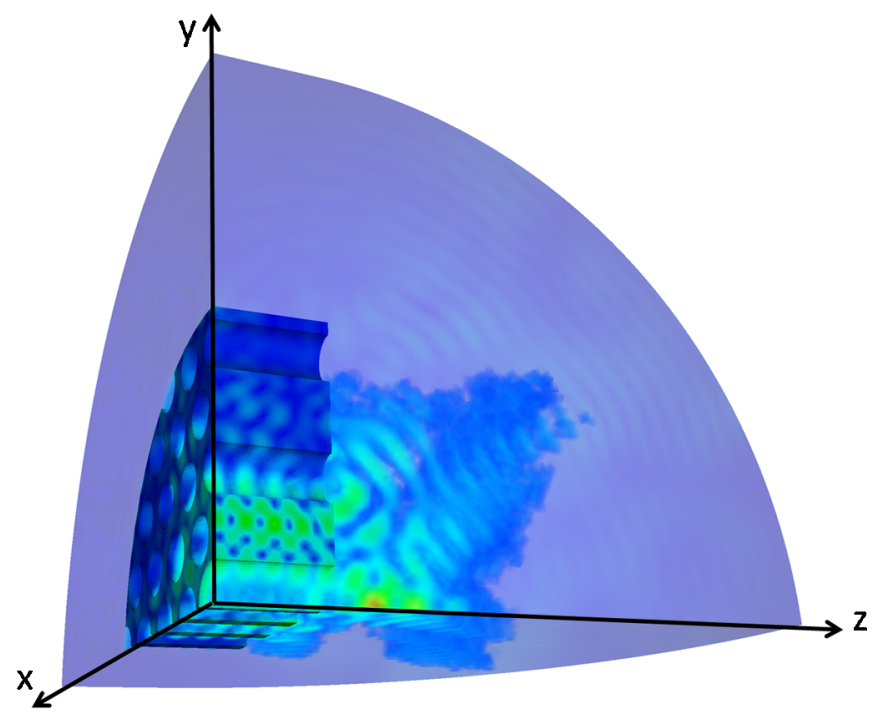

FIG. 7. Snapshot of the radiated electric field from the propagation of the defect mode in the fiber. In the simulation, the length of the fiber slab is a little longer than $2 \lambda$ and the radius of the sphere enclosing the simulation model is $10 \lambda$.

rise time is used similar to that in the previous section. In contrast to the previous case of wave propagation in a uniform PBG slab, the smooth propagation of the electromagnetic fields of the defect mode will be intercepted at the PBG-vacuum interface. Part of the power carried by the defect mode will be reflected and the rest will transmit out the right-hand boundary of the PBG and radiate into free space. The radiated electromagnetic waves will be terminated at the surrounding spherical surface for the simulation.

A snapshot of the electric field amplitude at steady state is shown in Fig. 7. It can be seen that the radiated field propagates in a nonuniform manner with power confined in several cones with certain solid angles. The detail of the radiated distribution will be discussed later. The electric field inside the PBG fiber shows the modulation of the forward traveling wave as a result of the reflection at the PBG-vacuum interface. The diffracted wave has a concentrated high-field region shown in red which is located at a distance of about one wavelength in the forward direction along the symmetry axis $(z)$ of the fiber. This is due to the constructive interference of the uniform field of the accelerating mode scattering from the exit of the circular defect aperture. At this position, the field distributions on the plane perpendicular to the $z$ axis are shown in Fig. 8 with the electric field still showing strong longitudinal polarization components. Notice that there are other components of the radiation field that are barely visible in Fig. 7, and we will discuss these further in Fig. 10.

Figure 9 shows the power radiated out to the spherical surface of the computational volume as well as that reflected back into the PBG fiber. Positive and negative values of the power again indicate that power is flowing (a)

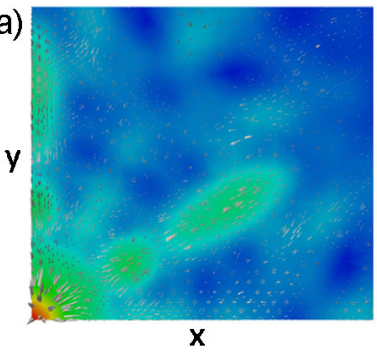

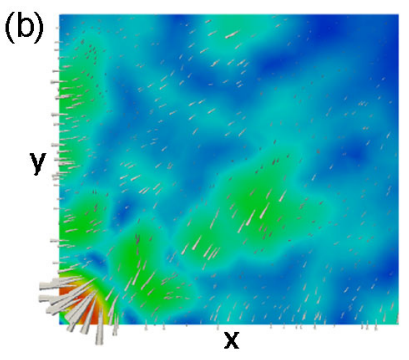

FIG. 8. The amplitudes of (a) the electric field and (b) the magnetic field at a distance of about one $\lambda$ downstream from the PBG-vacuum interface. The cones represent the field vectors and their sizes scale as the magnitudes of the fields.

outward and inward through a surface, respectively. The time evolution of the instantaneous power at the output spherical surface shows that the electric and magnetic fields of the radiated waves are not in phase (compare to Fig. 6), indicating that interference effects from the diffracted waves at this distance are still important and preclude the establishment of a far-field radiation pattern. The positive values of the instantaneous power at the input port mean that some power is reflected and propagates backward into the PBG fiber. The averaged radiated and reflected power is found to be about $81 \%$ and $19 \%$, respectively.

Figure 10 shows the radiation pattern on the spherical surface, looking from the longitudinal axis of the PBG fiber. This represents one quarter of the forward hemisphere. Two prominent peaks in the Poynting flux can be seen on the surface. The location of each peak is defined by two angles $(\theta, \phi)$, where $\theta$ is the angle between the longitudinal $z$ axis and the line joining the peak location and the origin defined at the fiber-vacuum interface, and $\phi$

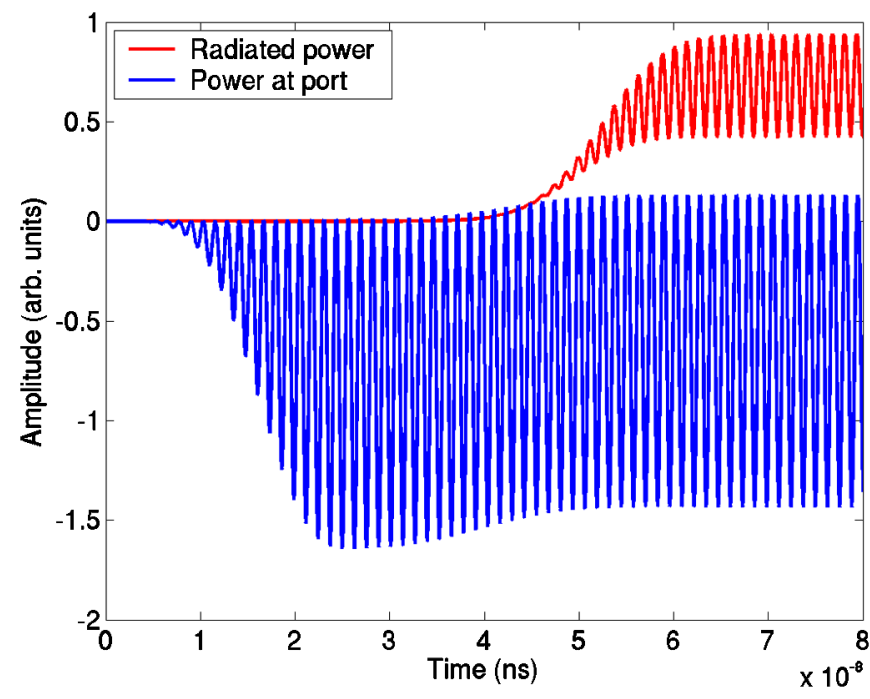

FIG. 9. Radiated power (red) and power transmitted at the input port (blue) for the propagation of the defect mode at the entrance of the fiber. 


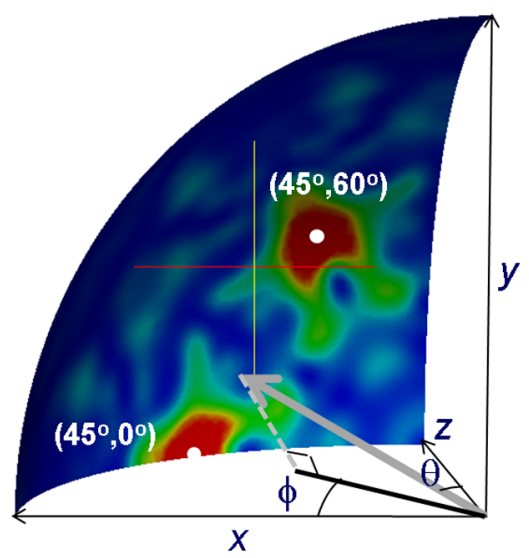

FIG. 10. Radiated power pattern in the forward quarter spherical surface (with a radius of $8 a$ or about $10 \lambda$ ) from defect mode propagation in the fiber, showing angular position $(\theta, \phi)$ of the peak field regions.

defined by the projection on the transverse $x-y$ plane of the PBG fiber. Both peaks are located at $\theta=45^{\circ}$ from the longitudinal, symmetry $z$ axis and the two transverse angles $\phi$ are at $0^{\circ}$ and $60^{\circ}$ with respect to the $x$ axis (Fig. 10).

\section{B. Far-field radiation pattern}

Following the procedure in the previous subsection, one can obtain the far-field radiation pattern on the spherical surface by using a forward hemisphere with a large radius $\gg \lambda$. However, the computational domain will become very big and the calculation will be very time consuming. To circumvent this problem, we extract the fields on the plane of the fiber-vacuum interface and use them as excitation sources in calculating the far-field radiation. Figure 11 shows the electric and magnetic fields on this plane. The field patterns at this plane preserve most of those of the defect mode shown in Fig. 3.

The far-field radiation is determined using Huygen's principle [14] and obtained through the following procedure. The source plane is divided into a number of patches using a rectangular grid. Within each grid, the current
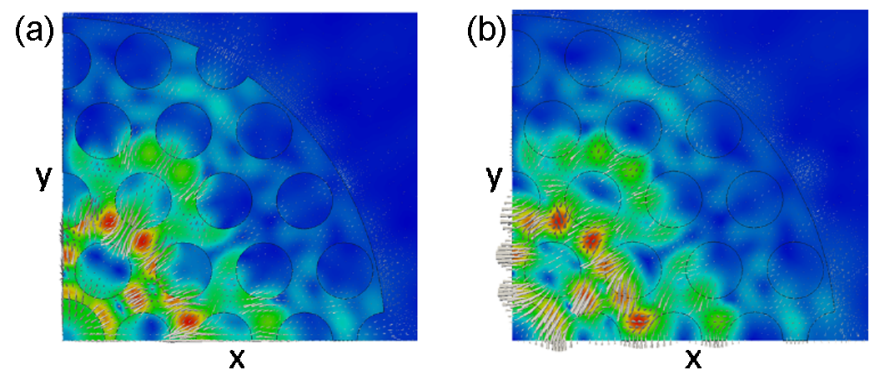

FIG. 11. The amplitudes of (a) the electric field and (b) the magnetic field at the PBG-vacuum interface. The cones represent the field vectors and their sizes scale as the magnitudes of the fields. sources are calculated using the electric and magnetic field vectors obtained from the T3P simulation. Since only a quarter of the structure is simulated in T3P, the source plane representing the full model is obtained by reflecting appropriately the fields through the symmetry planes. The display surface of the far-field radiation is chosen to be a forward hemisphere with its origin at the defect center. The distant spherical surface is also divided into a number of patches, the dimensions of which are set to $0.2 \lambda$. The far-field radiation in each patch is determined by all the patches in the source plane through the propagation of the electromagnetic radiation in free space. Thus, the computation for the far field on the whole surface scales as the product of the numbers of the patches in the source plane and radiation surface. A MATLAB propagator code [15] was used for the calculation, and the choice of $0.2 \lambda$ resolution for the surface patches is found to be sufficient to reveal the radiation pattern within a reasonable amount of computational time.

Figure 12 shows the Poynting flux of the far-field radiation on the hemispherical surface as a function of the radius $R$ of the hemisphere, scaled to the same size for ease of display. The radiation pattern changes drastically from $R=5 \lambda$ to $R=20 \lambda$ and reaches a steady configuration at $R=80 \lambda$. It should be noted that the radiation pattern at $10 \lambda$ is very similar to that obtained by direct T3P simulation where the radius of the spherical surface enclosing the computation domain is $10 \lambda$. Using the source fields determined by a different code [16] for PBG mode calculation and propagating these with the MATLAB code shows similar radiation patterns as those shown in Fig. 12, verifying qualitatively the correctness of our calculations. As shown from Fig. 12, the far-field radiation pattern has a sixfold symmetry and is localized in the high-field regions (indicated by red color) on the downstream surface. Remembering that the $z$ axis is in the forward direction perpendicular to the source plane, the centers of the highfield regions are located at $\theta=45^{\circ}$ from the $z$ axis, and spaced at $60^{\circ}$ in the azimuth $\phi$. This angular dependence agrees well with that obtained from direct simulation using T3P (Fig. 10). To estimate the spatial extent of a high-field region on the surface as shown in the three-dimensional plot at $R=80 \lambda$ in Fig. 13, the boundary of the region is defined by the Poynting flux value dropped by two $e$ foldings of the maximum. It is found that the six high-field regions together contain $75 \%$ of the radiated power within $33 \%$ of the total surface area.

Figure 14 shows the variations of the Poynting flux along the longitude and latitude passing through the peak of a high-field region on the forward hemisphere with radius $80 \lambda$. It can be seen that the shape along the longitude is very close to a Gaussian and the variation along the latitude is rather linear in nature. Figure 15 shows the shape of the high-field region along the longitude and latitude for different hemisphere radii $R$ from $10 \lambda$ to $120 \lambda$. The shape along 

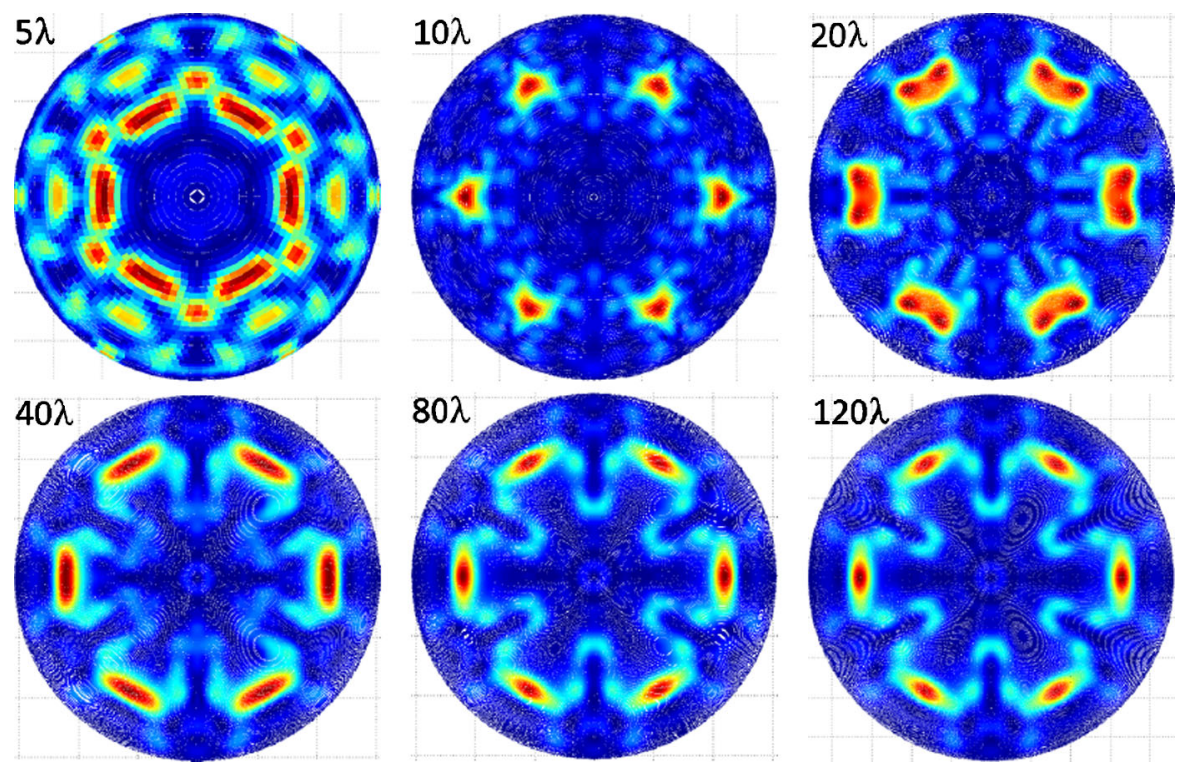

FIG. 12. Projection of Poynting flux from the surface of the forward hemisphere onto a circular disk (scaled) as a function of the hemisphere radius at $5 \lambda, 10 \lambda, 20 \lambda, 40 \lambda, 80 \lambda$, and $120 \lambda$.

the longitude is always narrower than that along the latitude. Along the longitude, the width of the Gaussian-like shape gets narrower as $R$ increases until it reaches a steady state above $80 \lambda$. Along the latitude, the shape is rather Gaussian-like at $R=10 \lambda$, after which it broadens out with a double-humped profile and changes back to a broad peak

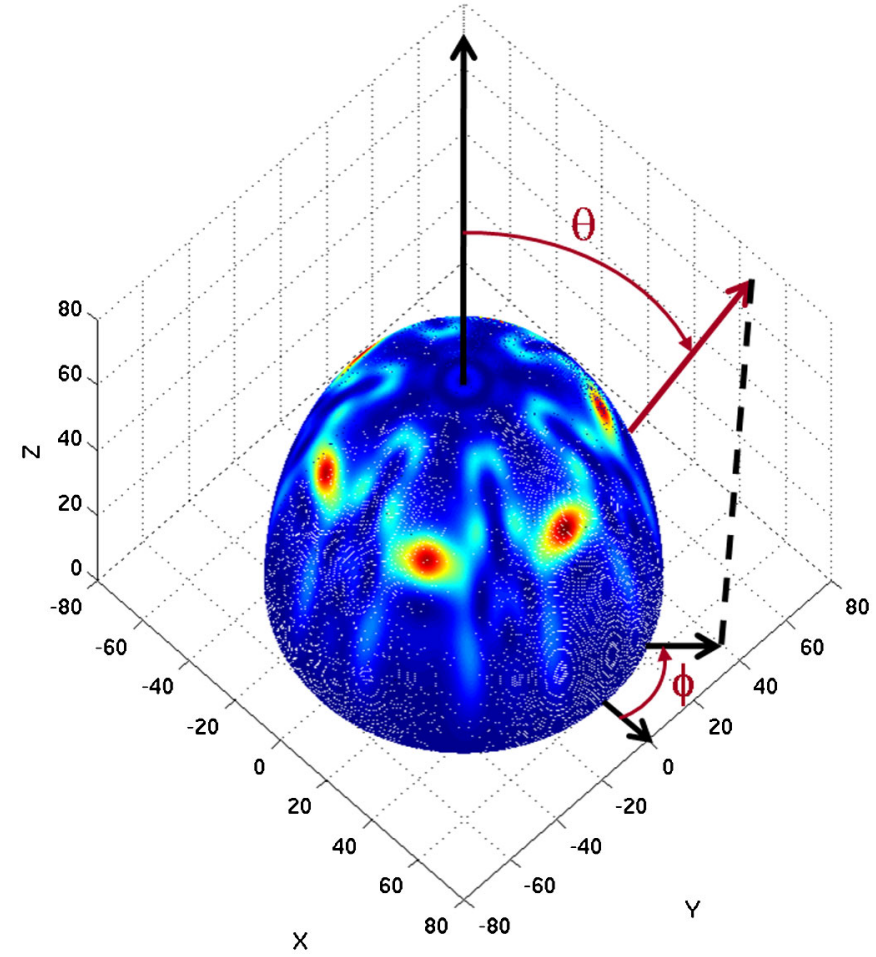

FIG. 13. Poynting flux on the surface of the forward hemisphere with radius $80 \lambda$. The $z$ axis is normal to the source plane, and the $x$ and $y$ axes define the source plane. with linear drops on both sides when $R$ is greater than $80 \lambda$. This illustrates the complicated interference effects contributed by the current sources as one moves away from them. A brief word about radiation and coupling effects in such fiber may be useful here before proceeding because the two are clearly related. For the modes of concern here, losses from the sides and ends need to be considered and,

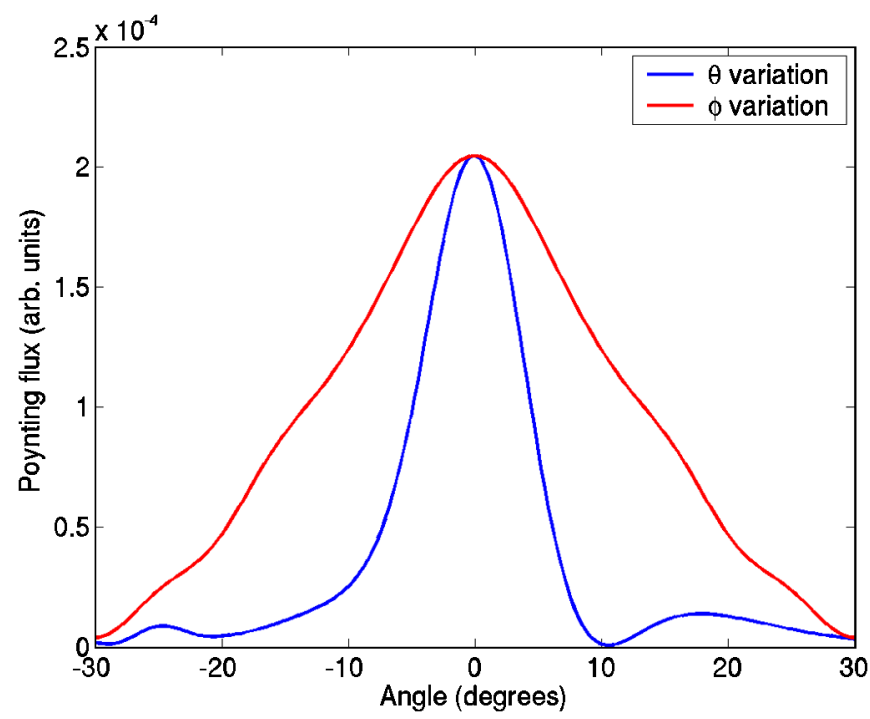

FIG. 14. Variations of Poynting flux along the longitude and latitude passing the peak of a high-field region on the forward hemisphere with radius $80 \lambda$. The variation along the latitude covers a range of $60^{\circ}$, the angle of rotation symmetry of the radiation pattern. The variation along the longitude spans from $0^{\circ}-90^{\circ}$ but is predominately confined to about $30^{\circ}$ and is shifted by $45^{\circ}$ so that its peak coincides with that along the latitude for easy comparison. 

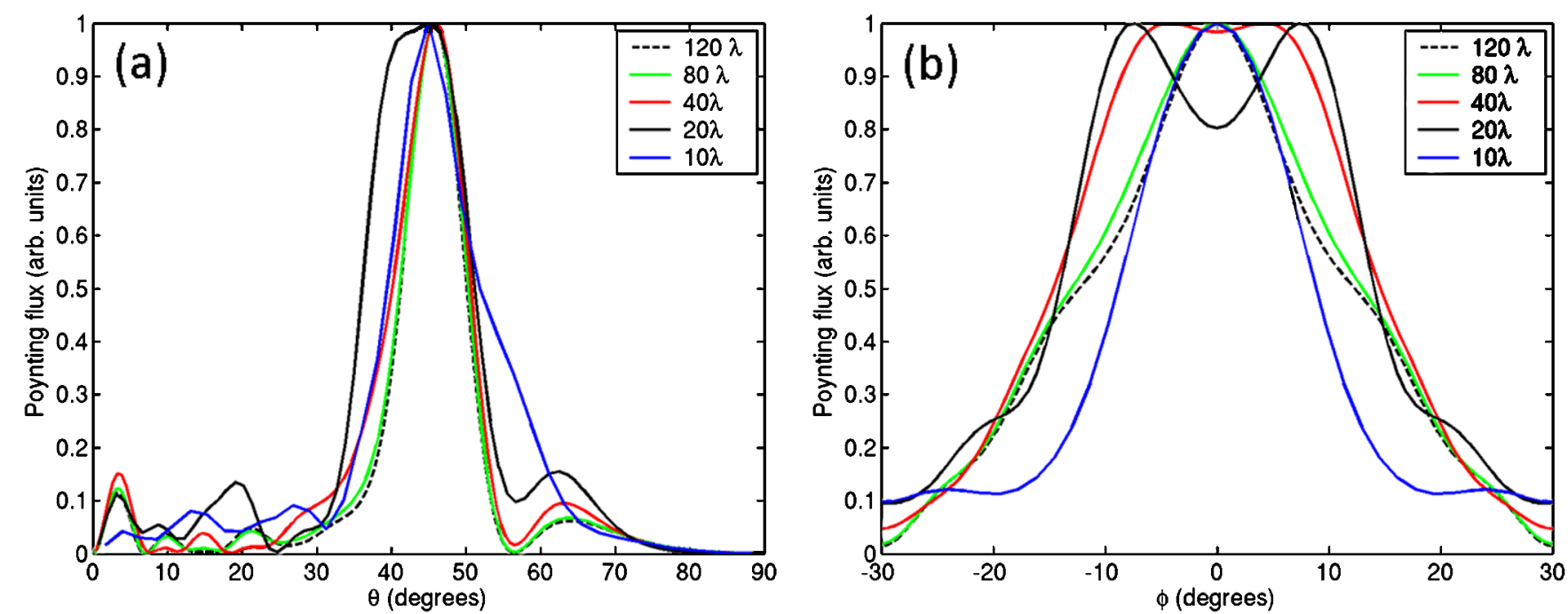

FIG. 15. The shapes of a high-field region along (a) the longitude and (b) the latitude for different hemisphere radii (in units of $\lambda$ ).

(a)

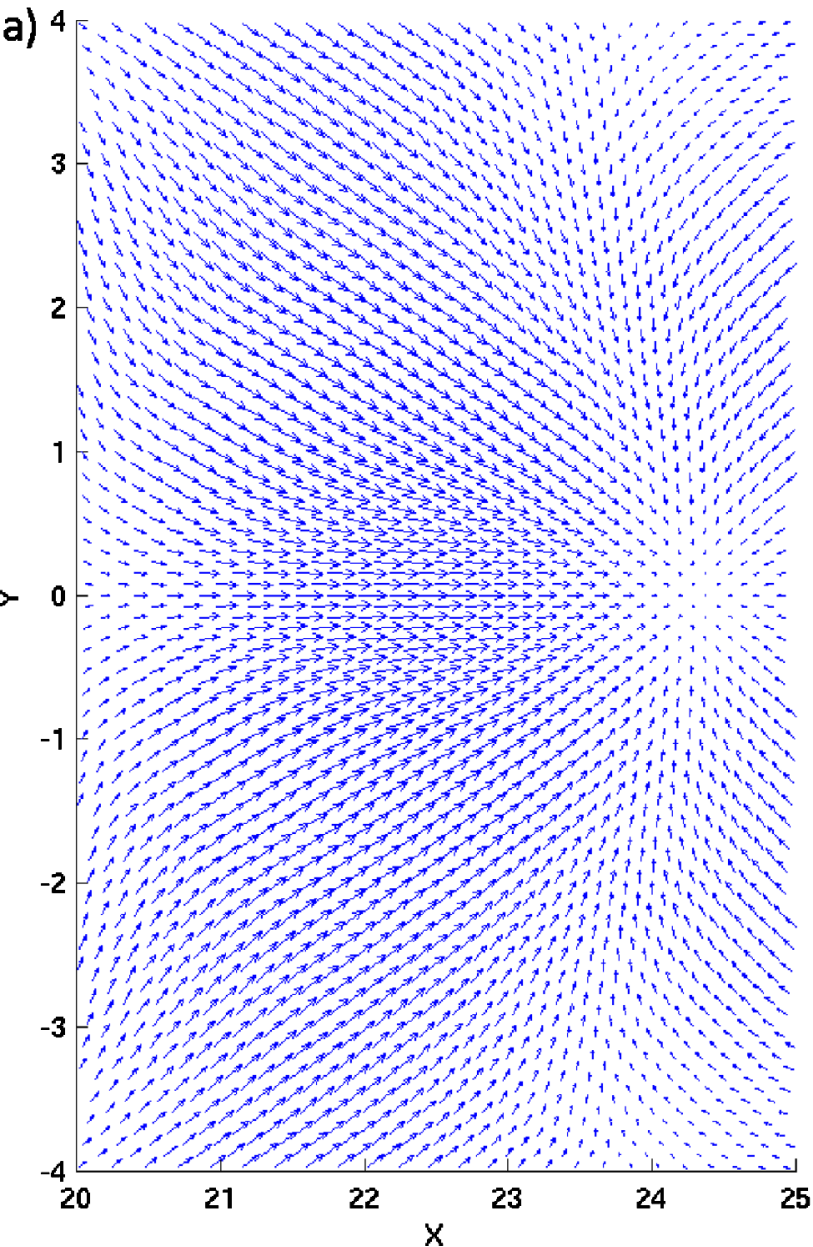

(b)

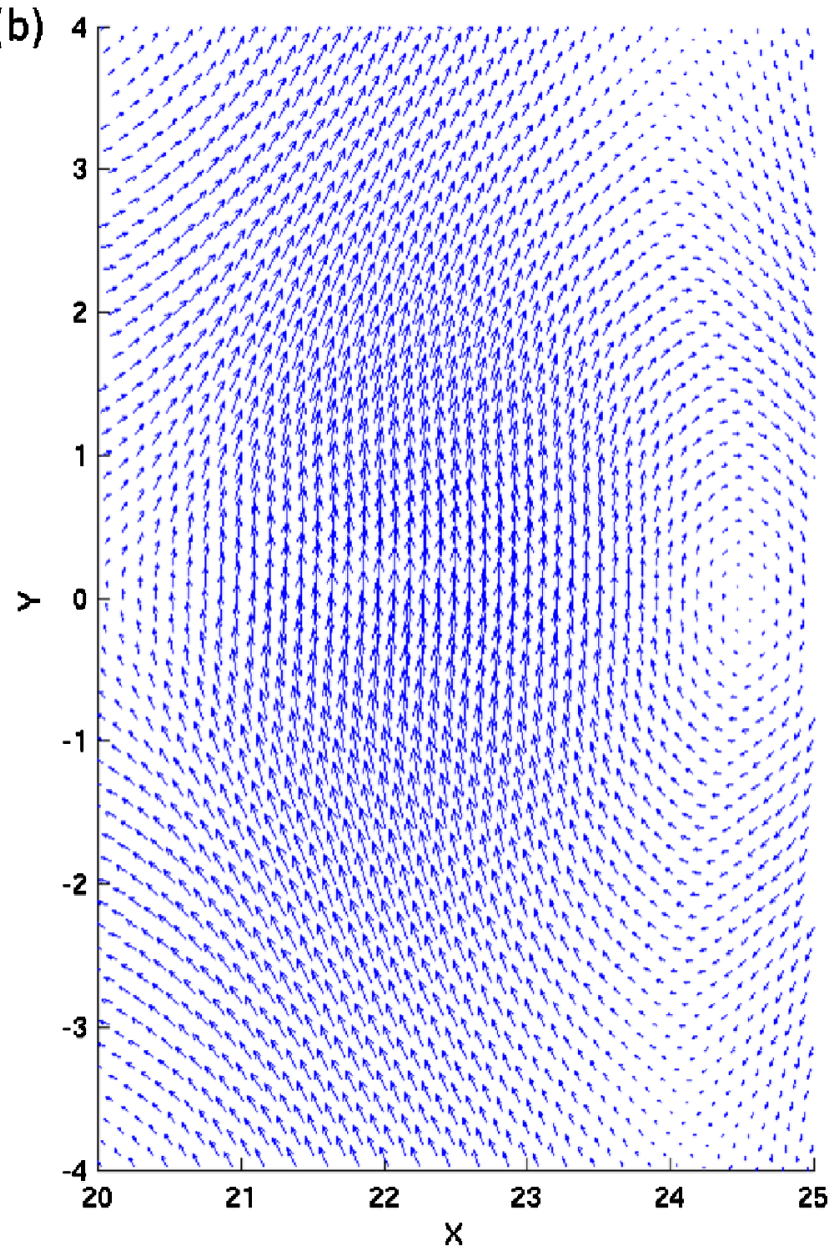

FIG. 16. Polarizations of electric (a) and magnetic (b) fields in the far right high-field region $\left(\phi=0^{\circ}, \theta=45^{\circ}\right)$ of the far-field radiation shown in Fig. 12 for the hemisphere radius at $80 \lambda$. 
while these have different solutions, they are both defined by the basic hexagonal symmetry of the lattice. Losses from both sides and ends typically show sixfold symmetry and will be addressed in a subsequent paper where perturbations of the pure hexagonal lattice symmetry about the defect are broken in various periodic and aperiodic ways to achieve coupling.

Figure 16 shows the polarizations of the electric and magnetic fields of the high-field region located at azimuthal position $\phi=0^{\circ}$ in Fig. 13. The far-field vectors have been projected from the spherical surface on which they were calculated onto the $x-y$ plane for ease of visualization. The electric and magnetic fields within this region are seen to be predominantly polarized in the $x$ and $y$ directions, respectively. The field configurations of the other five high-field regions are identical to those of Fig. 16 under a sequence of $60^{\circ}$ rotations about the $z$ axis. The far-field radiation of this mode is therefore seen to consist of six distinct lobes with radially polarized electric and azimuthally polarized magnetic fields, which diverge from the origin at a polar angle of approximately $45^{\circ}$.

Matching of external laser light into the fiber in an experimental setup might therefore be accomplished using either an array of six linearly polarized laser beams, whose polarizations are rotated relative to each other in increments of $60^{\circ}$, or by nonparaxial focusing of a single laser mode with the requisite radial polarization and hexagonal symmetry. For example, a TEM $_{03}$ Laguerre-Gaussian laser mode closely resembles the far-field radiation pattern of Fig. 16 in both symmetry and polarization. This mode can be formed by combination of the more common HermiteGaussian laser modes [17,18]. In addition, commercial techniques [19] are available for production of an azimuthally symmetric, radially polarized $\mathrm{TEM}_{01}^{*}$-like mode, which represents an easily obtained experimental mode profile for coupling to the structure. Calculation of the coupling efficiencies of various schemes will be the subject of a future communication.

\section{CONCLUSION}

Dielectric fibers driven by lasers represent a promising medium for acceleration of charged particles, capable of sustaining much higher field gradient than traditional microwave structures, and of being manufactured inexpensively using well-established industrial techniques [20]. An optimized fiber geometry was proposed in Ref. [3] that uses the central defect of a hollow-core photonic band-gap fiber to produce a single confined mode with an accelerating gradient that is uniform over the beam channel. We have extended this work, using a combination of frequency and time-domain codes to calculate the propagation of radiation in the fiber and the radiation of the confined mode propagating from the end of the fiber into free space. Because, to our knowledge, this is the first work of its kind on this problem, we have shown in some detail how one approaches the solution and verifies the result.

The far-field pattern obtained, as detailed in Figs. 13-16, represents the ideal free-space mode profile which if propagated in reverse would produce optimal coupling to the accelerating mode of the fiber. A phase 2 grant has recently been awarded by the U.S. Department of Energy to build prototypes of this structure at operating wavelengths from 1.5 to $2 \mu \mathrm{m}$ [21], and a separate program is underway to couple laser light into and demonstrate acceleration of a test beam in these and other structures [4]. Our present results should therefore be highly informative for these experimental efforts. They also provide an operational template by which to conduct similar calculations both for other laser-dielectric accelerator schemes and for PBG fibers in use by the telecom industry.

\section{ACKNOWLEDGMENTS}

We would like to thank Eric Colby for many useful discussions and use of his MATLAB code, and Greg Schussman for help with visualization. The work was supported by the U.S. DOE Contract No. DE-AC0276SF00515. The work used the resources of NCCS at ORNL which is supported by the Office of Science of the U.S. DOE under Contract No. DE-AC05-00OR22725, and the resource of NERSC at LBNL which is supported by the Office of Science of the U.S. DOE under Contract No. DEAC03-76SF00098.

[1] M. Rosing and W. Gai, Phys. Rev. D 42, 1829 (1990).

[2] A. Mizrahi and L. Schachter, Phys. Rev. E 70, 016505 (2004).

[3] X.E. Lin, Phys. Rev. ST Accel. Beams 4, 051301 (2001).

[4] R. J. England et al., in Advanced Accelerator Concepts Workshop (Santa Cruz, 2008), AIP Conf. Proc. No. 1086 (AIP, New York, 2009), p. 550.

[5] ACE3P: https://slacportal.slac.stanford.edu/sites/ard_public/bpd/acd/Pages/Default.aspx.

[6] L.-Q. Lee et al., Technical Report No. SLAC-PUB-13529, 2009.

[7] A.E. Candel et al., in Proceedings of the International Computational Accelerator Physics Conference, Chamonix Mont-Blanc, France, 2006 [http://accelconf .web.cern.ch/AccelConf/ICAP06/PAPERS/ WEMPMP03.PDF].

[8] N. Kroll, D. R. Smith, and S. Schultz, in Advanced Accelerator Concepts (Port Jefferson, 1992), AIP Conf. Proc. No. 279 (AIP, New York, 1993), p. 197.

[9] E. Colby et al., IEEE Trans. Nucl. Sci. 49, 2857 (2002).

[10] J. E. Spencer, Int. J. Mod. Phys. A 18, 2903 (2003).

[11] R. Noble and J. Spencer, in Proceedings of the 2007 Particle Accelerator Conference, Albuquerque, New Mexico (IEEE, Albuquerque, New Mexico, 2007).

[12] K. Leung and Y. Liu, Phys. Rev. Lett. 65, 2646 (1990).

[13] B. C. Stuart et al., J. Opt. Soc. Am. B 13, 459 (1996). 
[14] L. Diaz and T. Millagan, Antenna-Engineering Using Physical Optics (Artech House, Inc., London, 1996).

[15] E. Colby (private communication).

[16] B. Kuhlmey, CUDOS MoF Utilities for Micro-Structured Optical Fibres, University of Sydney, Australia [http:// sydney.edu.au/science/physics/cudos/].
[17] J. Courtial and M. J. Padgett, Opt. Commun. 159, 13 (1999).

[18] L. Allen et al., Phys. Rev. A 45, 8185 (1992).

[19] M. Stalder and M. Schadt, Opt. Lett. 21, 1948 (1996).

[20] M. Nielsen et al., Opt. Express 12, 1372 (2004).

[21] http://www.er.doe.gov/sbir/Awards_Abstracts/ARRA/ ARRAPhaseII.html. 